\title{
DOUBLE-RESONANCE METHODS IN GASES, WITH APPLICATION TO OCS
}

\author{
P. HOYNG*, H. A. DIJKERMAN and G. RUITENBERG** \\ Fysisch Laboratorium der Rijksuniversiteit te Utrecht, Nederland
}

Received 12 May 1971

\section{Synopsis}

We present a systematic derivation of and a few corrections to the theory on double resonance given by Autler and Townes. We discuss the range of validity of this theory and show its relation with the theory formulated by Di Giacomo. In addition, a critical comparison of both theories with experimental results is given. In these experiments the $J=1,2$ l-type doublets of OCS have been studied: the microwave absorption spectrum is determined in the presence of a radio-frequency field. The influence of the parameters of the microwave and the radio-frequency field on the fine structure of the microwave absorption spectrum is studied. The agreement between theories and experiment is satisfactory. The $J=1$ and $J=21$-doublet spacings of OCS are found to be $12.78 \pm 0.01 \mathrm{MHz}$ and $38.17 \pm 0.01 \mathrm{MHz}$, respectively.

1. Introduction. The subject of double resonance and three-level maser action has been explored both experimentally and theoretically during the last fifteen years $\left.{ }^{1-6}\right)$. Application to the spectroscopy of polar gases has been described in several papers ${ }^{7-9}$ ). Generally, power is fed from two microwave oscillators into the sample, and only in a few cases the combination of microwave and radio-frequency power has been used $\left.{ }^{10,11}\right)$. The density-matrix formalism provides the proper theoretical tool for description of the dynamic behaviour of a system of molecules under the influence of external forces. It is relatively easy to find a formal expression for the energy-absorption coefficient $\alpha(\omega)$ in terms of the parameters of the two electromagnetic fields, the hamiltonian of a single molecule, the number density of the gas and the temperature. In order to proceed, two typical cases $A$ and $B$ are introduced as to the values of the parameters of the electromagnetic fields. For each case the theory is then developed by making the appropriate approximations, using the formal expression for $\alpha(\omega)$ as a

* Present address: Laboratorium voor Ruimteonderzoek der Rijksuniversiteit te Utrecht, Nederland.

** Present address: AKZO Research Laboratories, Arnhem, Nederland. 
starting point. The theory for case $\mathrm{A}$ amounts to a generalization of the theory of Autler and Townes ${ }^{10}$ ) while that for case B turns out to be equivalent with the theory given by $\mathrm{Di}$ Giacomo ${ }^{4}$ ). These are the contents of section 2. It should be remarked that the theory developed here is not general in the sense that (1) we explicitely make use of the specific relative position of the four levels as given in fig. 1 and cq. (10a, b) and (2) we assume that the two electric fields are parallel. In section 3 we report the results of our doubleresonance experiments on the $J=1,2$ l-type doublets of OCS, obtained with a conventional Stark-modulation spectrometer. We mention a few complications of experimental origin that must be allowed for in the interpretation of the results.

2. Theory. The density-matrix formalism is used to describe a system of molecules under the influence of one or more electromagnetic fields. The total hamiltonian for a single molecule will be:

$$
H(t)=H_{0}+H_{1}(t)=H_{0}-\mu_{z} E \cos \omega t-\mu_{z} E_{0} \cos \omega_{0} t,
$$

where $H_{0}$ is the hamiltonian for the free molecule. The two linearly polarized electric fields are taken to be parallel and directed along the $z$ axis. $E, \omega$ are the parameters of the microwave field and $E_{0}, \omega_{0}$ those of the radio-frequency field. The quantity of interest is the energy-absorption coefficient for the microwave field, $\alpha(\omega)$ :

$$
\alpha(\omega)=-\frac{4 \pi N \omega}{c} \operatorname{Im} \chi(\omega)
$$

where $N$ is the number density of the gas, $c$ the velocity of light. The dimension of $\alpha(\omega)$ is $\mathrm{cm}^{-1} \cdot \chi(\omega)$ is the susceptibility, given by

$$
\left\langle\mu_{\boldsymbol{z}}\right\rangle=\operatorname{Re}\left[\chi(\omega) E \mathrm{e}^{\mathrm{i} \omega t}\right],
$$

(see, e.g., Kubo ${ }^{12}$ ). Finally, the time-dependent expectation value of the dipole-moment operator $\mu_{z}$ is given by

$$
\left\langle\mu_{z}\right\rangle=\operatorname{Tr} \rho \mu_{z},
$$

where $\rho$ is the density matrix. In this paper we assume that the simplest model of pressure broadening, as has been given by Karplus and Schwinger ${ }^{13}$ ), is sufficient. In their treatment, the density matrix $\rho(t)$ is averaged over the time of the last collision. The state of the molecule immediately after the collision is given by the density matrix $\rho_{0}(t)$ describing thermal equilibrium with the instantaneous values of the fields. Successive collisions are assumed to be uncorrelated. These assumptions are not unreasonable if we have "impact conditions" (i.e. large average distance between the molecules; duration of collision sufficiently short and collision sufficiently strong). 
An equation is derived ${ }^{13}$ ) for the difference $D(t)$ between the density matrix $\rho(t)$ and that for instantaneous thermal equilibrium $\rho_{0}(t)$ :

$$
\left(\frac{\partial}{\partial t}+\frac{1}{\tau}\right) D(t)=\frac{1}{\mathrm{i} \hbar}[H(t), D(t)]-\frac{\partial}{\partial t} \rho_{0}(t),
$$

where

$$
D(t)=\rho(t)-\rho_{0}(t) ; \rho_{0}(t)=\mathrm{e}^{-\beta H(t)} / \operatorname{Tr} \mathrm{e}^{-\beta H(t)} \quad \text { and } \quad \beta=(k T)^{-1} .
$$

$\tau$ is the relaxation time for restoring instantaneous thermal equilibrium; in this paper $\tau$ is considered to be a phenomenological parameter. On the microscopic scale $\tau$ represents the average time between two successive collisions. The stationary solution of eq. (5) is:

$$
D(t)=-\int_{0}^{\infty} \mathrm{d} \alpha \mathrm{e}^{-\alpha / \tau} U(t \mid t-\alpha) \dot{\rho}_{0}(t-\alpha) U(t-\alpha \mid t) .
$$

The dot indicates the partial derivative with respect to time. $U\left(t \mid t_{0}\right)$ is the evolution operator, defined by $U\left(t \mid t_{0}\right)=M(t) M^{-1}\left(t_{0}\right)$ where $M$ is any non-singular solution of

$$
\dot{M}(t)=\frac{1}{\mathrm{i} \hbar} H(t) M(t) .
$$

The evolution operator contains all dynamical information of the system between collisions and it is unique: if $M_{1}$ and $M_{2}$ are two non-singular solutions of eq. (7) then therc exists a non-singular time-independent linear operator $C$ such that $M_{2}=M_{1} C$, and one has:

$$
\begin{aligned}
& U_{2}\left(t \mid t_{0}\right) \overline{\overline{\mathrm{D}}} M_{2}(t) M_{2}^{-1}\left(t_{0}\right) \\
& \quad=M_{1}(t) C\left[M_{1}\left(t_{0}\right) C\right]^{-1}=M_{1}(t) M_{1}^{-1}\left(t_{0}\right) \overline{\overline{\mathrm{D}}} U_{1}\left(t \mid t_{0}\right) .
\end{aligned}
$$

Under prevailing experimental conditions, the parameters $\mu_{z} E / k T, \mu_{z} E_{0} / k T$ that occur in $\beta H_{1}(t)$ are always very small ( $\$ 10^{-6}$ ), where $\mu_{z}$ now momentarily stands for a typical matrix element of the transitions concerned. Accordingly one makes a series expansion of $\rho_{0}(t)$ in terms of $\beta H_{1}(t)$ and keeps only the first two terms ${ }^{13}$ ):

$$
\begin{aligned}
& \rho_{0}(t)=\frac{\mathrm{e}^{-\beta\left[H_{0}+H_{1}(t)\right]}}{\operatorname{Tr} \mathrm{e}^{-\beta\left[H_{0}+H_{1}(t)\right]}} \approx \rho^{(0)}\left[1-\int_{0}^{\beta} \mathrm{e}^{\lambda H_{0}} H_{1}(t) \mathrm{e}^{-\lambda H_{0}} \mathrm{~d} \lambda+\ldots\right] \\
& \approx \rho^{(0)}\left[1+\left(E \mathrm{e}^{\mathrm{i} \omega t}+E_{0} \mathrm{e}^{\mathrm{i} \omega_{0} t}\right) \int_{0}^{\beta} \mathrm{e}^{\lambda H_{0}} \mu_{z} \mathrm{e}^{-\lambda H_{0}} \mathrm{~d} \lambda\right],
\end{aligned}
$$

where $\rho^{(0)}=\mathrm{e}^{-\beta H_{0}} / \operatorname{Tr} \mathrm{e}^{-\beta H_{0}}$ is the normal density matrix describing thermal 
equilibrium with respect to the unperturbed hamiltonian $H_{0}$. Differentiating eq. (8) with respect to time and inserting the result in eq. (6), one finds an expression for the absorption coefficient for the microwave field with the aid of eqs. (4), (3) and (2). After some rearranging we find:

$$
\begin{aligned}
\alpha(\omega) & =\frac{4 \pi N \omega^{2}}{c} \operatorname{Re} \int_{0}^{\infty} \mathrm{d} \alpha \mathrm{e}^{-\alpha / \tau-\mathrm{i} \omega \alpha} \\
& \times \int_{0}^{\beta} \mathrm{d} \lambda \operatorname{Tr}\left[\rho^{(0)} \mathrm{e}^{\lambda H_{0}} \mu_{z} \mathrm{e}^{-\lambda H_{0}} \overline{U(t-\alpha \mid t) \mu_{z} U(t \mid t-\alpha)}\right] .
\end{aligned}
$$

Thus, the problem of the calculation of $\alpha(\omega)$ is reduced to the calculation of $U$, that is, to finding a solution $M$ from eq. (7). The bar in eq. (9) indicates a time average. The explicit $t$ dependence of $U(t-\alpha \mid t)$ is caused by our assumption that the external fields are strictly monochromatic waves with well-defined phases. In fact, the relative phase of the two fields will drift in an uncontrollable way within the resolution time of the experimental apparatus. So one should take an average over the phases, but this is equivalent to the time average indicated in eq. (9). Actually, the complete expression for $\alpha(\omega)$ contains three separate terms instead of one as given in eq. (9). However, the time-average operation reduces the contribution of the two extra terms to zero. A more elegant treatment ${ }^{14,5}$ ) has circumvented this time-averaging procedure.

To be able to make more explicit statements, we shall now adhere to a four-level system analogous to the $J=1,2$ l-type doublets of OCS (fig. 1). Allowed transitions are indicated and we assume each level to be nondegenerate ${ }^{\dagger}$. Another example of fig. 1 may be found in the $\mathrm{K}$ doublets of

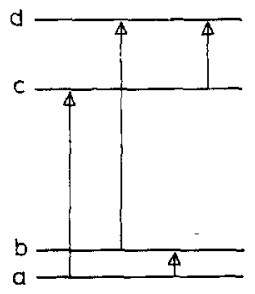

Fig. 1

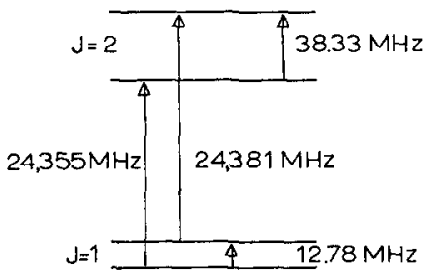

Fig. 2

Fig. 1. The four-level system used in this paper. Allowed transitions are indicated and the relative values of the transition frequencies are given in eq. (10a).

Fig. 2. An example of such a four-level system, consisting of the $J=1,2$ l-type doublets of OCS.

$\dagger$ Because both fields $E, E_{0}$ are parallel, we have the general selection rule $\Delta M=0$. Each $M$ system may thus be treated independently and one simply adds the various contributions afterwards. 
slightly asymmetric tops. The characteristics of fig. 1 are conveniently defined as follows:

$$
\begin{aligned}
& \left.\begin{array}{l}
H_{0}|p\rangle=\varepsilon_{p}|p\rangle \\
\varepsilon_{q}-\varepsilon_{p}=\hbar \omega_{p q}
\end{array}\right\} \quad p, q=a, b, c \text { or } d, \\
& \omega_{a c} \approx \omega_{b d} \gg \omega_{a b} \approx \omega_{c d}, \\
& \left\langle a\left|\mu_{z}\right| c\right\rangle=\left\langle b \mu_{z} \mid d\right\rangle ; \quad\left\langle a\left|\mu_{z}\right| d\right\rangle=\left\langle b \mu_{z} \mid c\right\rangle=0 .
\end{aligned}
$$

The $\varepsilon_{p}$ are the four energy levels concerned and the $\omega_{p q}$ are possible transition frequencies. We shall choose the frequencies $\omega, \omega_{0}$ near resonance, anticipating conditions as they were in experiments with OCS:

$$
\begin{array}{lll}
\omega \approx \omega_{a c} & \text { or } \quad \omega \approx \omega_{b d} & \text { (microwave field) } \\
\omega_{0} \approx \omega_{a b} & \text { or } \quad \omega \approx \omega_{e d} & \text { (radio-frequency field) }
\end{array}
$$

We assume that it is allowed to neglect the existence of any other energy level. The operators in the various equations (1) through (9) are then $4 \times 4$ matrices. As is well known, a formal solution $M$ of eq. (7) can be written 14, 15):

$$
M(t)=Y(t) \mathrm{e}^{\mathrm{i} A t}, \quad \text { with } \quad Y(t)=\sum_{k l} P k l \mathrm{e}^{-\mathrm{i}\left(k \omega+l \omega_{0}\right) t}
$$

and $M$ can be chosen unitary: $M M^{\dagger}=1$.

The $P^{k l}$ are time-independent $4 \times 4$ matrices and $A$ is a $4 \times 4$ diagonal matrix with real, time-independent elements. $A$ and the $P^{k l}$ can, at least in principle, be determined by substitution of eq. (11) in eq. (7), taking $H(t)$ from eq. (1). However, if $H(t)$ contains two or more frequencies (as it does here) it is impossible to find the general solution.

We now introduce a classification of the field strengths $E, E_{0}$. First we define a quantity $b$ with the dimension of frequency:

$$
b=p \varepsilon / 2 \hbar,
$$

where $\varepsilon$ is a typical field strength and $p$ is a typical dipole matrix element. A field strength $\varepsilon$ may be classified as weak, saturating or strong according to:

$$
\begin{array}{ll}
b \ll 1 / \tau: & \text { weak-field strength } \varepsilon, \\
b \approx 1 / \tau: & \text { saturating-field strength } \varepsilon, \\
b \approx \omega: & \text { strong-field strength } \varepsilon,
\end{array}
$$

where $1 / \tau$ and $\omega$ stand for the linewidth and the frequency of a transition, respectively. This transition should be explicitly stated for the definition to make sense and moreover we should have $1 / \tau \ll \omega$ (as is usually the case). Now for the microwave field $E$ we shall take the transitions $a \rightarrow c$ or $b \rightarrow d$ and for the radio-frequency field $E_{0}$ the transitions $a \rightarrow b$ or $c \rightarrow d$. Since 
$\omega_{a b} \approx \omega_{c d}$ and $\omega_{a c} \approx \omega_{b a}$ and since all four dipole matrix elements are of the same order of magnitude, the classification (13) is unambiguous. We shall treat in detail the cases:

A. weak microwave field + strong radio-frequency field,

B. saturating microwave field + saturating radio-frequency field.

A case with a strong microwave ficld cannot occur since it is far beyond experimental possibilities: it would require about $1 \mathrm{MW} / \mathrm{cm}^{2}$ power input for the microwave field (take $\omega \approx 24 \mathrm{GHz}$ and $p \approx 0.7 \mathrm{D}$ ). Henceforth we use the abbreviations $r f$ for radio-frequency and $\mathrm{mf}$ for microwave frequency.

2.1. Case A. The absorption of a weak $\mathrm{mf}$ field is measured in the presence of a strong rf field. This case has been treated before by Autler and Townes ${ }^{10}$ ). In eq. (7) we may now neglect the microwave field in $H(t)$, and it reduces to:

$$
\dot{M}=\frac{1}{\mathrm{i} \hbar}\left[H_{0}-\mu_{z} E_{0} \cos \omega_{0} t\right] M .
$$

The $(a, b)$-and the $(c, d)$-level systems will not be coupled by the if field $E_{0}$ and they can be treated completely independently. That means that $M$ can be decomposed:

$$
M=\left[\begin{array}{cccc}
* & * & & 0 \\
* & * & & \\
0 & * & *
\end{array}\right]=\left[\begin{array}{cc}
M^{a b} & 0 \\
& \\
0 & M c d
\end{array}\right] .
$$

Each of the $2 \times 2$ submatrices $M^{a b}$ and $M^{c a}$ should satisfy eq. (14) and this equation can be solved easily with the help of the usual Floquet theorem to which formula (11) reduces if only one external frequency is involved. Our notations are partly based on those of Autler and Townes, who prove that $M^{a b}$ can be written as:

$$
M^{a b}-\left[\begin{array}{lc}
\sum_{n} a_{2 n} \mathrm{e}^{-2 \mathrm{i} n \omega_{0} t} & -\sum_{n} a_{2 n+1} \mathrm{e}^{(2 n+1) \mathrm{i} \omega_{0} t} \\
\sum_{n} a_{2 n+1} \mathrm{e}^{-(2 n+1) \mathrm{i} \omega_{0} t} & \sum_{n} a_{2 n} \mathrm{e}^{2 \mathrm{i} n \omega_{0} t}
\end{array}\right]\left[\begin{array}{cc}
\mathrm{e}^{\mathrm{i} \lambda_{a} t} & 0 \\
0 & \mathrm{e}^{\mathrm{i} \lambda_{b} t}
\end{array}\right] .
$$

The summations run over all integers.

$$
\lambda_{a}=-\varepsilon_{a} / \hbar+b_{a} L_{a} ; \quad \lambda_{b}=-\varepsilon_{b} / \hbar-b_{a} L_{a},
$$

with

$$
b_{a}=\frac{E_{0}}{2 \hbar}\left\langle a\left|\mu_{z}\right| b\right\rangle \quad[c f . \text { eq. (12)]. }
$$

$L_{a}$ and $a_{n}$ are, respectively, eigenvalue and eigenvector of a real symmetric 
infinite matrix of tridiagonal shape (see also the appendix and, e.g., ref. 14):
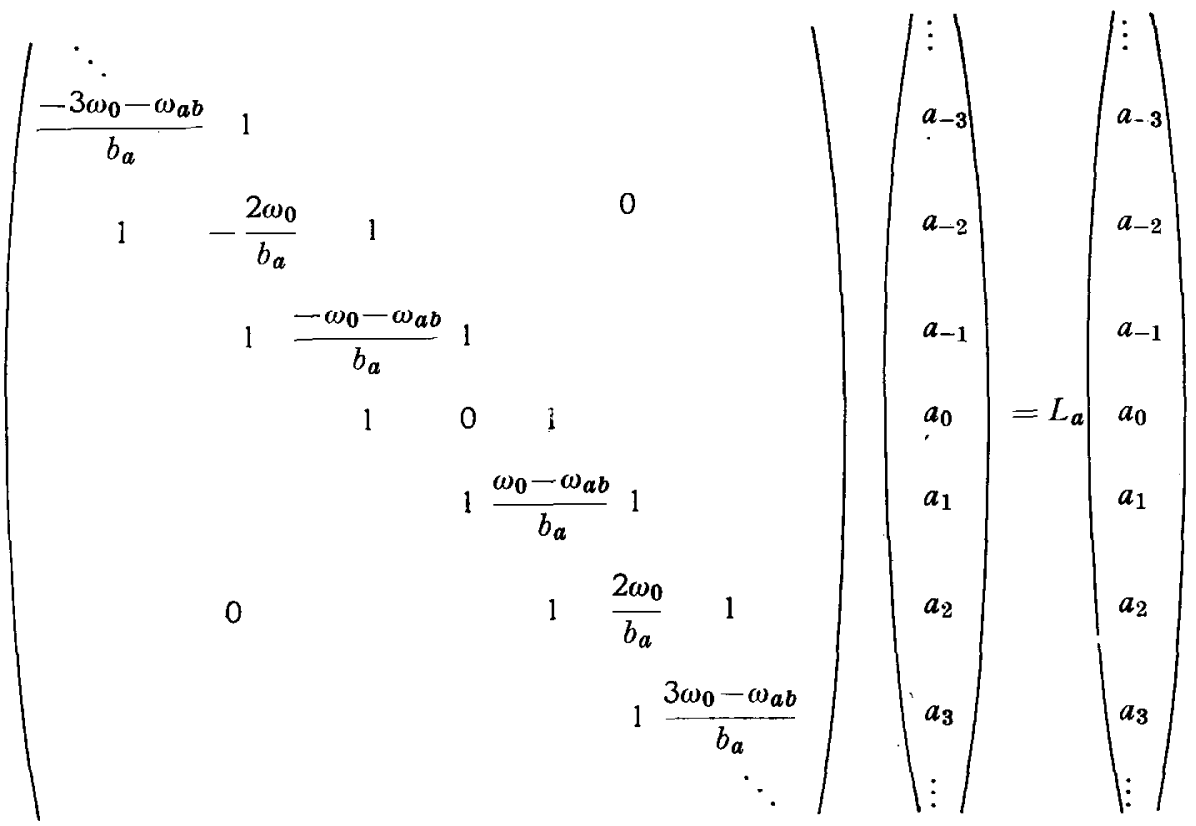

Eq. (18) has (countably) infinitely many solutions $L_{a},\left\{a_{n}\right\}$. If, however, $L_{a},\left\{a_{n}\right\}$ is a solution of eq. (18) then also:

$$
\left\{\begin{array} { l } 
{ L _ { a } ^ { \prime } = L _ { a } - \frac { 2 s \omega _ { 0 } } { b _ { a } } } \\
{ a _ { n } ^ { \prime } = a _ { n + 2 s } }
\end{array} \text { and } \left\{\begin{array}{l}
L_{a}^{n}=-L_{a}-\frac{\omega_{a b}-(2 s+1) \omega_{0}}{b_{a}} \\
a_{n}^{\prime \prime}=(-1)^{n+1} a_{2 s-n+1}
\end{array},\right.\right.
$$

for any integer s. Eq. (19) is a direct consequence of the simple periodicity properties of the tridiagonal matrix. It can be proved that eq. (19) defines all eigenvalues and eigenvectors of eq. (18). (Essentially, one uses the methods as described in ref. 16.) Since any non-singular solution $M$ should give the same results, our final formulae should be invariant for the transformations (19). In the limit $E_{0} \rightarrow 0$, i.e. $b_{a} \rightarrow 0$, we infer from eq. (14): $M \rightarrow \exp \left(-H_{0} t / \mathrm{i} \hbar\right)$, that is:

$$
M^{a b} \rightarrow\left(\begin{array}{cc}
\mathrm{e}^{-i \varepsilon_{a} t / \hbar} & 0 \\
0 & \mathrm{e}^{-i \varepsilon_{b} t / \hbar}
\end{array}\right) \quad \text { for } \quad E_{0} \rightarrow 0 .
$$

From the formulae (16) through (20) one can easily prove that there exists exactly one solution $L_{a},\left\{a_{n}\right\}$ with:

$$
\left.\begin{array}{l}
b_{a} L_{a} \rightarrow 0 \\
a_{n} \rightarrow \delta_{n, 0}
\end{array}\right\} \quad \text { for } \quad b_{a} \rightarrow 0 .
$$

Finally we choose $M$ to be unitary. This imposes the following condition 
on the $\left\{a_{n}\right\}$ :

$$
\sum_{n} a_{n} a_{n+2 s}=\delta_{0, s}
$$

For $s \neq 0$ this equation is merely a restatement of the well-known fact that eigenvectors of a symmetric matrix corresponding to different eigenvalues are orthogonal. For $s=0 \mathrm{eq}$. (22) states that we must choose eigenvectors of eq. (18) normalized to unity.

Autler and Townes ${ }^{10}$ ) solved eq. (18) with the laborious method of continued fractions. In addition, we have treated eq. (18) numerically in order to prove the correctness of the approximations made by Autler and Townes. In view of the relations (19) and (21) we have worked with the eigenvalue $L_{a}$ with the smallest absolute value; from eq. (19) we deduce an upper limit: $\left|L_{a}\right| \lesssim \omega_{0} / b_{a}$. This eigenvalue turned out to be very insensitive to the procedure of truncation of the infinite matrix to a finite $k \times k$ matrix, which is necessary of course in order to handle eq. (18) on a computer. For example, if $\omega_{0} \approx \omega_{a b}$ and $b_{a} \lesssim \omega_{a b}$ then $k \approx 15$ is already sufficient for an accurate calculation of $L_{a}$. Once $L_{a}$ is known it is a standard routine to determine the (truncated) normalized eigenvector $\left\{a_{n}\right\}$. The structure of this eigenvector is such that the row

$$
a_{0}, a_{1}, a_{-1}, a_{2}, a_{-2}, a_{3}, a_{-3}, \ldots
$$

exhibits a very fast (roughly exponential) convergence to zero. This again confirms the fact that we do not introduce any serious error with the truncation procedure. The results of the numerical computations are given in figs. 4 and 5 . The solution for $M^{c d}$ is now rather trivial. In eqs. (16) through (22) one replaces everywhere:

$$
a_{n} \rightarrow c_{n} ; \quad a \rightarrow c ; \quad b \rightarrow d .
$$

This defines the new quantities $b_{c}, L_{c}$ and $\left\{c_{n}\right\}$. The calculation of $L_{c},\left\{c_{n}\right\}$ and their properties are the same in every detail as explained above for $L_{a},\left\{a_{n}\right\}$. From now we assume that $L_{a},\left\{a_{n}\right\}$ and $L_{c},\left\{c_{n}\right\}$ are known, either as approximate analytical expressions in $b_{a}, \omega_{a b}, \omega_{0}$ or $b_{c}, \omega_{c d}, \omega_{0}$ as given by Autler and Townes or as sets of numerically calculated values for different values of $b_{a}, \omega_{a b}, \omega_{0}$ or $b_{c}, \omega_{c d}, \omega_{0}$. Since now $M$ has been solved for from eq. (14), we can construct $U\left(t \mid t_{0}\right)$ and substitute it in eq. (9) to find $\alpha(\omega)$; the calculations are straightforward but lengthy. The general result is not given here; we just mention that the invariance requirements to the transformations (19) are satisfied. Instead, we introduce some simplifications due to the specific geometry of the level system [relations (10a, b)] and we also omit all terms that are resonant at

$$
\omega=0, \omega \approx \pm \omega_{a b}, \omega \approx \pm \omega_{c d}, \omega \approx-\omega_{a c} \text { and } \omega \approx-\omega_{b a}
$$


We are then left with:

$$
\begin{aligned}
\alpha(\omega) & =\frac{4 \pi N \omega^{2}}{c} m^{2} r \\
& \times \sum_{p=-\infty}^{\infty}\left\{\frac{A_{p}^{2}}{\left[\left(\omega-\omega_{a d}\right)+(2 p+1) \omega_{0}-b_{a} L_{a}-b_{c} L_{c}\right]^{2}+\frac{1}{\tau^{2}}}\right. \\
& +\frac{A_{p}^{2}}{\left[\left(\omega-\omega_{b c}\right)-(2 p+1) \omega_{0}+b_{a} L_{a}+b_{c} L_{c}\right]^{2}+\frac{1}{\tau^{2}}} \\
& +\frac{C_{p}^{2}}{\left[\left(\omega-\omega_{a c}\right)-2 p \omega_{0}-b_{a} L_{a}+b_{c} L_{c}\right]^{2}+\frac{1}{\tau^{2}}} \\
& \left.+\frac{C_{p}^{2}}{\left[\left(\omega-\omega_{b d}\right)+2 p \omega_{0}+b_{a} L_{a}-b_{c} L_{c}\right]^{2}+\frac{1}{\tau^{2}}}\right\}
\end{aligned}
$$

where

$$
A_{p}=\sum_{n=-\infty}^{\infty}\left(a_{2 n} c_{2 p-2 n+1}-a_{2 p-2 n+1} c_{2 n}\right), \quad C_{p}=\sum_{n=-\infty}^{\infty} a_{n} c_{n+2 p}
$$

are complicated functions of $\omega_{0}, b_{a}, b_{c}, \omega_{a b}$ and $\omega_{c a}$. In cq. (23) $m$ is a dipolcmoment matrix element and $r$ is a thermal factor:

$$
\begin{aligned}
m & =\left\langle a\left|\mu_{z}\right| c\right\rangle=\left\langle b\left|\mu_{z}\right| d\right\rangle \quad[c f . \text { eq. }(10 b)] ; \\
r & =\frac{\mathrm{e}^{-\beta \varepsilon_{1}}-\mathrm{e}^{-\beta \varepsilon_{2}}}{\varepsilon_{2}-\varepsilon_{1}} \frac{1}{Z} .
\end{aligned}
$$

$r$ contains the partition function $Z=\operatorname{Tr} \mathrm{e}^{-\beta H_{0}} ; \varepsilon_{1}$ stands for $\varepsilon_{a}$ or $\varepsilon_{b}$, and $\varepsilon_{2}$ for $\varepsilon_{c}$ or $\varepsilon_{d}$ [due to (10a) it does not really matter which combination is chosen: in case of OCS $r$ remains constant within relative changes of $1 \%$ ]. There is in fact an additional simplification, if $\omega_{a b}$ and $\omega_{c d}$ are not simultaneously near resonance. With the relations (35) and (36) from ref. 10 we can prove that we may now neglect $b_{c} L_{c}$ against $b_{a} L_{a}$ or vice versa:

$$
\begin{array}{ll}
\left|b_{c} L_{c}\right| \ll\left|b_{a} L_{a}\right| & \text { if } \omega_{0} \approx \omega_{a b}, \\
\left|b_{c} L_{c}\right| \gg\left|b_{a} L_{a}\right| & \text { if } \omega_{0} \approx \omega_{c d} .
\end{array}
$$

Because the external fields are near resonant and because in OCS we have $\omega_{c d}=3 \omega_{a b}$, we can always use this inequality. In the limit of a very weak rf field we have $b_{a}, b_{c} \rightarrow 0$, and from eq. (21):

$$
b_{a} L_{a}, b_{c} L_{c} \rightarrow 0 ; A_{p} \rightarrow 0 ; C_{p} \rightarrow \delta_{0, p}
$$

and it is seen that eq. (23) reduces to the well-known Van Vleck-Weisskopf 

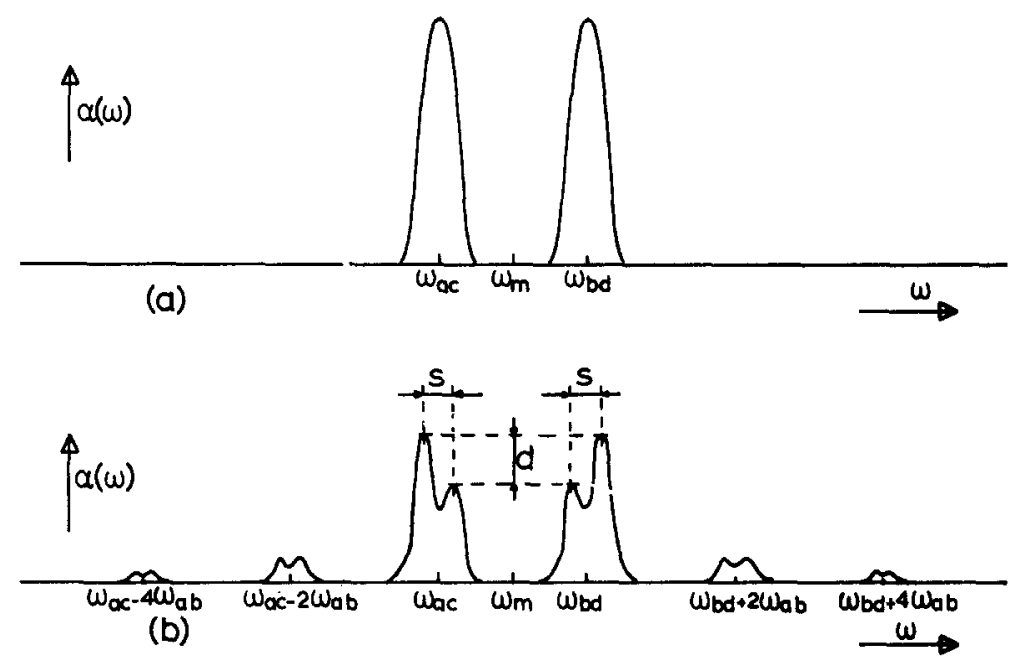

Fig. 3. Microwave absorption spectrum of the four-level system of fig. 1.

(a) Without rf field, $E_{0}=0$. (b) In the presence of a strong rf field $E_{0}$, for the case $\omega_{0} \approx \omega_{a b}$ and $\omega_{0}<\omega_{0}^{s}$. The figure defines the doublet splitting $s$ and the relative height $d$.

formula ${ }^{17}$ ) for the absorption coefficient and describes the absorption spectrum indicated in fig. $3 \mathrm{a}$.

After switching on the rf field, the spectrum is given by eq. (23) and it looks qualitatively as in fig. $3 \mathrm{~b}$ : it consists of a series of doublets with mutual separation of about $2 \omega_{0}$. The intensity decreases very rapidly to the left and right of the two main doublets, much more rapidly than indicated in fig. $3 \mathrm{~b}$. The splitting $s$ of a doublet is roughly proportional to the rf field strength $E_{0}$ (to $b_{a}$ if $\omega_{0} \approx \omega_{a b}$ and to $b_{c}$ if $\omega_{0} \approx \omega_{c d}$ ), and the relative height $d$ of the two lines in a doublet depends very strongly on $\omega_{0}$ at constant $E_{0}$ (therefore, adjustment of $d=0$ is a very sensitive experimental technique). The different lines in the spectrum correspond to different values of the integer $p$ in eq. (23). As indicated in fig. 3b, the absorption spectrum always satisfies the mirror property

$$
\alpha\left(\omega_{m}-\omega\right)=\alpha\left(\omega_{m}+\omega\right), \quad \text { where } \quad \omega_{m}=\left(\omega_{a c}+\omega_{b d}\right) / 2,
$$

provided $\omega \ll \omega_{m}$. In the appendix wc prove the sum rule

$$
\sum_{p}\left(A_{p}^{2}+C_{p}^{2}\right)=1
$$

which states that the sum of the top intensity of all lines in the absorption spectrum (fig. $3 \mathrm{~b}$ ) is constant, regardless the values of $E_{0}$ and $\omega_{0}$, if we discard the over-all factor $\omega^{2}$ in eq. (23).

Even for a strong rf field [in the sense of definition (13)] only the terms with $p=0$ in eq. (23) are important; we then have a doublet at $\omega \approx \omega_{a c}$ 
and $\omega \approx \omega_{b d}$, without side bands [however, for still higher field strength $E_{0}$ the terms with $|p|=1,2, \ldots$ from eq. (23) should become visible successively in the absorption spectrum. The existence of such a side-band effect has been shown experimentally by Chardon et al. $\left.{ }^{18}\right)$ ]. The condition for equal intensity $(d=0)$ is fulfilled for both doublets, if

$$
A_{0}^{2}=C_{0}^{2}, \quad \text { or }\left|\sum_{n} a_{n} c_{n}\right|=\left|\sum_{n}\left(a_{2 n} c_{1-2 n}-a_{1-2 n} c_{2 n}\right)\right| .
$$

Such doublets with $d=0$ will be called symmetrical and the frequency of the rf field at which this occurs will be called the frequency of symmetry. With the relations for $a_{n}$ and $c_{n}$ given by Autler and Townes $\left.{ }^{10}\right)$ eq. (27) can be reduced to a relation between the rf field strength $\left(b_{a}\right.$ or $\left.b_{c}\right)$ and the frequency of symmetry $\omega_{0}^{\mathrm{s}}$. The doublet splitting $s$ is also given:

$$
\begin{aligned}
& \omega_{0}^{\mathrm{s}}=\omega_{a b}+\frac{4 \mu_{c u} \omega_{a b}+\mu_{a b}\left(\omega_{c d} \cdots \omega_{a b}\right)}{\mu_{a b}\left(\omega_{c d}-\omega_{a b}\right)} \frac{b_{a}^{2}}{\omega_{a b}}+\mathcal{O}\left(b_{a}^{4}\right), \\
& s=2\left|b_{a}\right|-\left(\omega_{0}^{\mathrm{s}}-\omega_{a b}\right),
\end{aligned}
$$

for $\omega_{0} \approx \omega_{a b}$. We have used the obvious abbreviations:

$$
\mu_{p q}=\left\langle p\left|\mu_{z}\right| q\right\rangle ; \quad p, q=a, b, c \text { or } d .
$$

The case $\omega_{0} \approx \omega_{c d}$ is completely analogous: in eq. (28) one has to replace the indices $a$ by $c$ and $b$ by $d$. It is seen from eq. (28) that the case $\omega_{a b} \approx \omega_{c d}$

\begin{tabular}{|c|c|c|c|}
\hline \multicolumn{4}{|c|}{$\begin{array}{l}\text { Dipole matrix elements for the } J=1,2 \text { l-type } \\
\text { doublets of OCS in the } a, b, c, d \text { notation of fig } 1 . \\
\mu \text { represents the dipole moment of OCS; its value } \\
\left.\text { is taken to be } \mu=0.704 \mathrm{D}^{19}\right)\end{array}$} \\
\hline & $\mu_{a b}$ & $\mu_{c d}$ & $\mu_{a c}=\mu_{b d}$ \\
\hline $\begin{array}{l}M=0 \\
M= \pm 1 \\
M= \pm 2\end{array}$ & $\begin{array}{l}0 \\
\pm \frac{1}{2} \mu \\
-\end{array}$ & $\begin{array}{l}0 \\
\pm \frac{1}{6} \mu \\
\pm \frac{1}{3} \mu\end{array}$ & $\begin{array}{l}\mu \sqrt{\frac{1}{5}} \\
\mu \sqrt{\frac{3}{20}} \\
-\end{array}$ \\
\hline
\end{tabular}
should be treated separately. This can easily be done, but is not of our interest here.

TABLE I

We now specify eq. (28) for OCS. From table I we conclude that the $M=0$ four-level system has $b_{a}=b_{c}=0$ and it is thus not influenced at all by the rf field $E_{0}$. We discard it here (however, the presence of the $M=0$ level system does influence the measurements in the Stark-modulation spectrometer; see section 3). The $M=+1$ and the $M=-1$ level systems have (apart from a phase factor of -1 ) exactly the same dipole matrix elements and since the phase factor is unobservable, both systems behave identically with respect to the two electromagnetic fields (double-resonance experiments 
in which levels with $J>2$ are involved may exhibit a more complicated fine structure. The splitting for $M= \pm 1$ and $M= \pm 2$ need not be the same and as a result quartets or higher even multiplets are detected). We now deduce from eq. (28) for OCS with the aid of table I and the relation $\omega_{c a}=3 \omega_{a b}$ :

$$
\begin{aligned}
& \text { for } v_{0} \approx v_{a b}\left\{\begin{array}{l}
v_{0}^{\mathrm{s}}=v_{a b}+\frac{5}{3 v_{a b}} b^{2}+\mathcal{O}\left(b^{4}\right), \\
s=2 b-\frac{5}{3 v_{a b}} b^{2}+\mathcal{O}\left(b^{4}\right), \\
\text { and eliminating } b \text { from }(29 \mathrm{a}, \mathrm{b}): \\
v_{0}^{\mathrm{s}}=v_{a b}+\frac{1}{4}\left(\frac{5}{3 v_{a b}}\right) s^{2}+\frac{1}{8}\left(\frac{5}{3 v_{a b}}\right)^{2} s^{3}+\mathcal{O}\left(s^{4}\right),
\end{array}\right. \\
& \text { for } v_{0} \approx v_{c d}\left\{\begin{array}{l}
v_{0}^{\mathrm{s}}=v_{c d}-\frac{17}{9 v_{c d}} b^{2}+\mathcal{O}\left(b^{4}\right), \\
s=\frac{2}{3} b+\frac{17}{9 v_{c d}} b^{2}+\mathcal{O}\left(b^{4}\right), \\
\text { and eliminating } b \text { from }(30 \mathrm{a}, \mathrm{b}): \\
v_{0}^{\mathrm{s}}=v_{c d}-\frac{1}{4}\left(\frac{17}{v_{c d}}\right) s^{2}+\frac{1}{8}\left(\frac{17}{v_{c d}}\right)^{2} s^{3}+\mathcal{O}\left(s^{4}\right) .
\end{array}\right.
\end{aligned}
$$

In eqs. (29) and (30) we have converted to normal frequency units with $v=\omega / 2 \pi$ and with the use of $b_{c}=\frac{1}{3} b_{a}$ we expressed all $b_{a}, b_{c}$ dependence in the quantity $b=\left|b_{a}\right| 2 \pi \mid=\mu E_{0} / 4 h$, where $h$ is Planck's constant. The

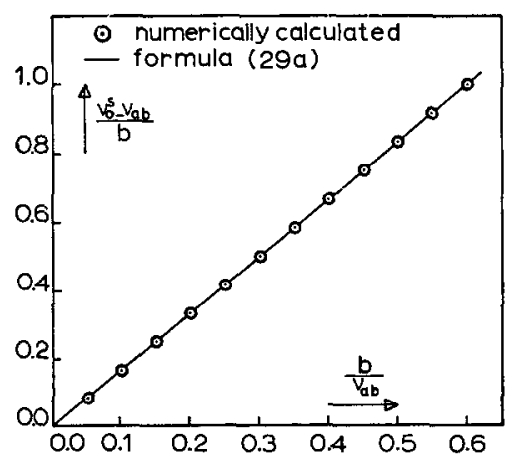

Fig. 4

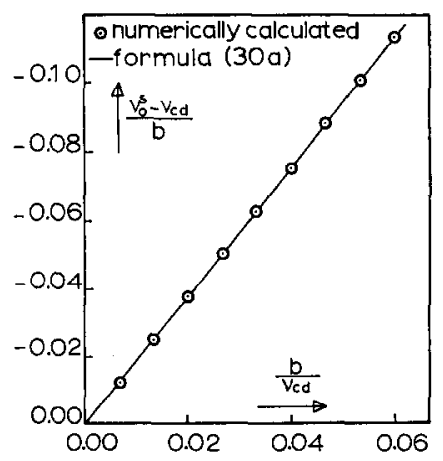

Fig. 5

Fig. 4. Comparison between theory presented in this paper and exact numerical computations for the frequency of symmetry $\nu_{0}^{8}$, for the case $\nu_{0} \approx v_{a b}$.

Fig. 5. The same as fig. 4 , but now $v_{0} \approx v_{c d}$. 
formulae (29c) and (30c) are given because in experiments the doublet splitting $s$ (rather than $b$ ) is directly measured. Eq. (28) has been derived from eq. (27) via quite a few approximations, that are not easily proved to be correct. Therefore, we have checked eqs. (29a) and (30a) by numerical treatment of the basic matrix equation (18) without making any further approximation except for the necessary truncation procedurc. The results are given in figs. 4 and 5. It is seen that eqs. (29a) and (30a) are good approximations within the range chosen for the parameter $b$. For larger values of $b$ deviations occur in both cases; tentatively, we impute this to the influence of the $b^{4}$ terms.

\section{Remarks}

a) One may readily check in eq. (28) that both $\mathrm{rf}$ transitions $a \rightarrow b$ and $c \rightarrow d$ determine the frequency of symmetry $\omega_{0}^{8}$, although the rf field is resonant with only one of them at most. At first glance, one would suspect that the non-resonant transition does not contribute at all, but the rf field being strong in the sensc of definition (13), transitions do take place; under these circumstances both rf transitions show a Bloch-Siegert shift 20 ), which is made visible by inducing transitions between both doublets with the aid of a weak mf field.

b) Apparently due to some mistake, the result of Autler and Townes corresponding to eq. (28) contains a $b_{a}^{3}$ term. However, the presence of odd powers of $b_{a}$ in eq. (28) for $\omega_{0}^{\mathrm{s}}$ would imply that $\omega_{0}^{\mathrm{s}}$ depends on the sign of the rf field. Such a dependence can be excluded using an argument similar to that following eq. (9). (A change in sign is equivalent to a phase change $\pi$.) However, if $b$ is eliminated between eqs. (29a) and (29b) and likewise between eqs. (30a) and (30b), we do get an $s^{3}$ term in eqs. (29c) and $(30 \mathrm{c})$.

c) The $J=2 \rightarrow 3, K_{-1}=2$ (vibrational ground state) transitions of the molecule methoxyethyne $\left(\mathrm{HC} \equiv \mathrm{COCH}_{3}\right)$ appeared to be a feasible experimental realization in the $\mathrm{mf}-\mathrm{rf}$ range of the above-mentioned splitting into quartets instead of doublets.

2.2. Case $B$. The absorption of a saturating mf field is measured in the presence of a saturating $\mathrm{rf}$ field. It is then not allowed anymore to neglect the $\mathrm{mf}$ field compared to the rf field in eq. (7), as we did in case A. A complete treatment with two external frequences instead of one as given in case $\mathrm{A}$ is unfeasible due to its computational complexity. Still, from the formal solution (11) we may construct the evolution operator $U(t \mid t-\alpha)$ :

$$
\begin{aligned}
& U(t \mid t-\alpha)=Y(t) \mathrm{e}^{\mathrm{i} \Lambda \alpha} Y(t-\alpha)^{\dagger}, \\
& U(t-\alpha \mid t)=U(t \mid t-\alpha)^{\dagger},
\end{aligned}
$$

and substitute it in eq. (6) for $D(t)$. It can be seen that all dependence of 
$D(t)$ on $\tau$ and $A$ is put into Lorentz-like denominators by integration over $\alpha$. $Y$ and $\dot{\rho}_{0}$ contribute with exponentials of the type $\exp \left[\mathrm{i}\left(k \omega+l \omega_{0}\right) t\right]$. This is a complicated way of staring the obvious fact that $D(t)$, being the stationary solution of eq. (5), must have the form:

$$
D(t)=\sum_{k l} D^{k l} \mathrm{e}^{\mathrm{i}\left(k \omega+l \omega_{0}\right) t} .
$$

This has bəen pointed out by Di Giacom ${ }^{4}$ ), who substituted eq. (32) into eq. (5) and subsequently solved for the time-independent $4 \times 4$ matrices $D k l$ with a perturbation method up to second order. This should give reasonable results for not too strong fields $E, E_{0}$. Once $D$ is known, the density matrix $\rho$ is known and with eqs. (2), (3) and (4) the absorption coefficient $\alpha(\omega)$ is found. Di Giacomo, however, treated a three-level system. But if the applied fields $E, E_{0}$ are of saturating strength at most [definition (13)] many-quantum effects can be neglected and only near-resonant transitions are important. For the four-level system of fig. 1 this means that if e.g. $\omega_{0} \approx \omega_{a b}$ and $\omega \approx \omega_{a c}$ the presence of the state $d$ may be neglected provided that all four transition frequencies are sufficiently different from each other (as is the case in OCS, fig. 2). The four-level system of fig. 1 may thus be treated here with di Giacomo's three-level theory ${ }^{\dagger}$. Anticipating experimental conditions, we assume in this section that the rf field is always tuned to the transition frequency $\omega_{a b}$, whereas the mf field will be turned to $\omega_{a c}$ or $\omega_{b d}$. This leads to considerable simplifications in Di Giacomo's general result. We are left with two cases, see figs. 6 and 7 .

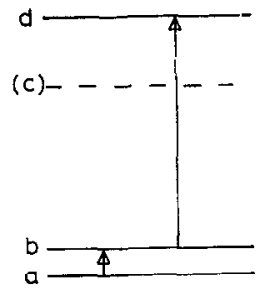

Fig. 6

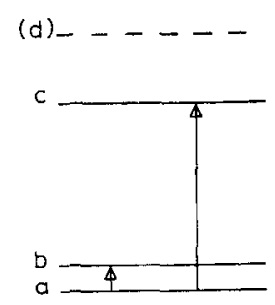

Fig. 7

Fig. 6. The four-level system from fig. 1 reduces effectively to a three-level system if $v_{a b}$ and $v_{c d}$ differ sufficiently and if the fields are at most saturating. If $v_{0} \approx v_{a b}$ and $v \approx v_{b d}$, the presence of the state $c$ can be neglected.

Fig. 7. The same as fig. 6 , but now $v_{0} \approx v_{a b}$ and $v \approx v_{a c}$; in this case the presence of the state $d$ can be disregarded.

+ As an objection it may be felt that it is not clear how Di Giacomo's perturbation method should be generalized unambiguously to third and higher order and that therefore, e.g., the proof of convergence cannot be relied upon. 
We introduce the dimensionless quantities

$$
\begin{aligned}
& \gamma_{a}=\tau^{2} b_{a}^{2}=\left(\frac{\tau E_{0} \mu_{a b}}{2 \hbar}\right)^{2} ; \\
& \gamma_{c}=\left(\frac{\tau E \mu_{a c}}{2 \hbar}\right)^{2}=\left(\frac{\tau E \mu_{b d}}{2 \hbar}\right)^{2},
\end{aligned}
$$

and because of the condition imposed on the field strengths we have: $\gamma_{a}, \gamma_{c} \lesssim 1$. We also define the occupation numbers

$$
f_{p}=\left\langle p\left|\rho^{(0)}\right| p\right\rangle ; \quad p=a, b, c \quad \text { or } \quad d .
$$

In the case of fig. $6 \mathrm{Di}$ Giacomo finds for the absorption coefficient $\alpha_{E}, E_{0}$ of the mf field tuned to $\omega_{b d}$ :

$$
\alpha_{E, E_{0}}=\frac{4 \pi N \tau}{\hbar c} \omega_{b d} \mu_{b d}^{2} \frac{3 \gamma_{a}\left(f_{a}-f_{b}\right)+\left(1+4 \gamma_{a}+\gamma_{c}\right)\left(f_{b}-f_{d}\right)}{\left(1+\gamma_{a}+\gamma_{c}\right)\left(1+4 \gamma_{a}+4 \gamma_{c}\right)} .
$$

From eq. (33) we derive the formula corresponding to fig. 7. First the labels that name the $a, b$-level system are interchanged in eq. (33), i.e. $f_{a} \leftrightarrow f_{b}$; $\omega_{b d} \rightarrow \omega_{a d}: \mu_{b d} \rightarrow \mu_{a d}$. Next we acknowledge that in fact $\varepsilon_{b}>\varepsilon_{a}$, so that $f_{b}-f_{a}=-\left(f_{a}-f_{b}\right)$ is a negative quantity. Finally one substitutes $d \rightarrow c$ (i.e. $d_{d} \rightarrow f_{c} ; \omega_{a d} \rightarrow \omega_{a c} ; \mu_{a d} \rightarrow \mu_{a c}$ ). We then have for the absorption coefficient $\alpha_{E, E_{0}}$ of the mf field $E$, turned to $\omega_{a c}$ :

$$
\alpha_{E, E_{0}}=\frac{4 \pi N \tau}{\hbar c} \omega_{a c} \mu_{a c}^{2} \frac{-3 \gamma_{a}\left(f_{a}-f_{b}\right)+\left(1+4 \gamma_{a}+\gamma_{c}\right)\left(f_{a}-f_{c}\right)}{\left(1+\gamma_{a}+\gamma_{c}\right)\left(1+4 \gamma_{a}+4 \gamma_{c}\right)} .
$$

We then infer from eq. (10a) the relations:

$$
\frac{f_{b}-f_{d}}{f_{a}-f_{b}} \approx \frac{f_{a}-f_{c}}{f_{a}-f_{b}} \approx \frac{\omega_{a c}}{\omega_{a b}} \gg 1
$$

With these relations eqs. (33) and (34) can be reduced to:

$$
\begin{aligned}
\alpha_{E, E_{0}} & =\frac{4 \pi N \tau}{\hbar c} \frac{1+4 \gamma_{a}+\gamma_{c}}{\left(1+\gamma_{a}+\gamma_{c}\right)\left(1+4 \gamma_{a}+4 \gamma_{c}\right)} \\
& \times \begin{cases}\mu_{b d}^{2} \omega_{b d}\left(f_{b}-f_{d}\right) & \omega=\omega_{b d} \\
\mu_{a c}^{2} \omega_{a c}\left(f_{a}-f_{c}\right) & \omega=\omega_{a c}\end{cases}
\end{aligned}
$$

Let $\alpha_{0}$ be the absorption coefficient of the $\mathrm{mf}$ field in the limit $E, E_{0} \rightarrow 0$ and let the function $F$ be defined by:

$$
F\left(\gamma_{a}, \gamma_{c}\right)=\frac{1+4 \gamma_{a}+\gamma_{c}}{\left(1+\gamma_{a}+\gamma_{c}\right)\left(1+4 \gamma_{a}+4 \gamma_{c}\right)} .
$$

Then eq. (35) can be written as:

$$
\alpha_{E, E_{0}}=F\left(\gamma_{a}, \gamma_{c}\right) \alpha_{0} \text {. }
$$




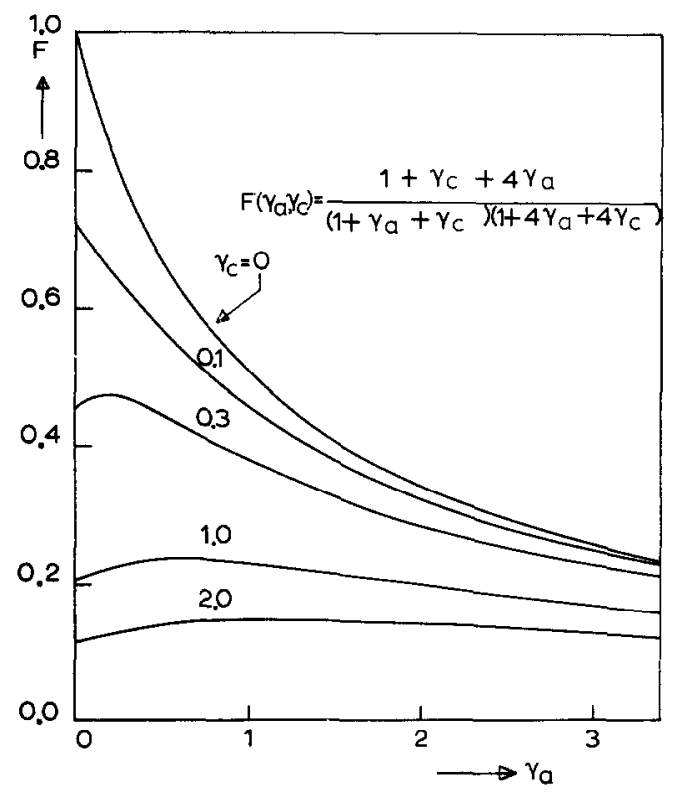

Fig. 8. Display of the function $F\left(\gamma_{a}, \gamma_{c}\right)$ defined in eq. (36). It is equal to the quotient of the absorption coefficient in the presence of at most saturating fields and the absorption coefficient in the limit of very weak fields.

The function $F$ in the latter equation is displayed in fig. 8. One may check that $F$ (and therefore $\alpha_{E, E_{0}}$ ) as a function of $\gamma_{a}$ reaches a maximum only if $\gamma_{c}>\frac{1}{8}$. This maximum is obtained when $\gamma_{a} \approx \frac{1}{2} \gamma_{c}$. The initial increase of $\alpha_{E, E_{0}}$ as a function of $\gamma_{a}$ can be considered as a partial cancellation of the saturation caused by the $\mathrm{mf}$ field. Finally, addition of the contributions of the two-level systems $M= \pm 1$ leaves eq. (37) unchanged; the $M=0$-level system does not contribute.

3. Experimental results. A conventional Stark-modulation spectrometer was used for the measurements on double-resonance effects. A simplified diagram of the apparatus is given in fig. 9. A frequency-stabilized klystron was used as a source of microwave radiation. The system allows for controlled variation as well as measurement of the frequency. The absorption cell was a waveguide with an insulated electrode for the Stark-modulation. The rf field was provided by an $\mathrm{rf}$ oscillator followed by an amplifier. The rf voltage and the Stark-voltage were brought together in an adding circuil as shown in fig. 9. The output was connected to the insulated electrode of the absorption cell.

3.1. Case A. The absorption of a weak $\mathrm{mf}$ field is measured in the presence of a strong $\mathrm{rf}$ field. The two $J=1,2$ 1-doublet lines of OCS were 


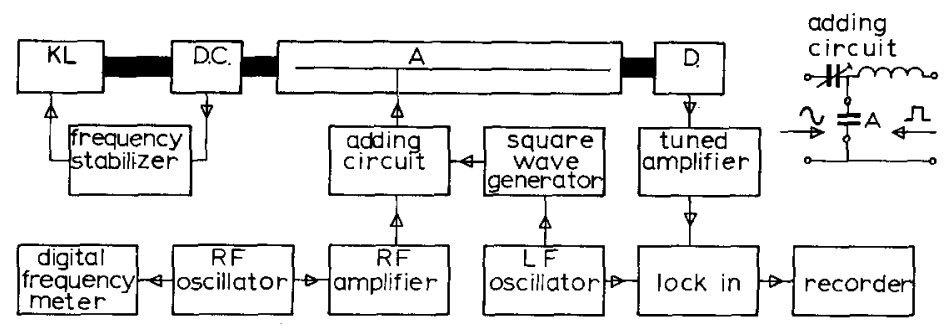

Fig. 9. Simplified diagram of the Stark-modulation spectrometer for double-resonance experiments. $\mathrm{KL}=$ klystron oscillator; $\mathrm{DC}=$ directional coupler; $\mathrm{A}=$ absorption cell with insulated electrode; $\mathrm{D}=$ detector.

used for these experiments. These lines were recorded as a function of the microwave frequency while the parameters of the rf field were kept constant. At sufficiently large amplitude of the rf signal the absorption lines were seen to be split into doublets as described in section 2.1 (fig. $3 \mathrm{~b}$ ). The frequency of the rf signal was adjusted until these doublets became symmetrical. This frequency, $v_{0}^{\mathrm{s}}$, is a function of the rf field strength $E_{0}$ according to eq. (29a). Since the determination of $E_{0}$ through measurement of the applied $\mathrm{rf}$ voltage was unreliable, $v_{0}^{\mathrm{s}}$ was plotted as a function of the doublet splitting $s$, as shown in fig. 10. From this figure it can be concluded that a linear relationship exists between $v_{0}^{8}$ and $E_{0}^{2}$ as given by eq. (29:a) up to strong rf-field strengths (the maximum rf-field strength used was about $50 \mathrm{~V} / \mathrm{cm}$. This corresponds to $b=\mu E / 4 h \approx 4.5 \mathrm{MHz}$ and $b / v_{a b} \approx 0.35$ ). The straight line in fig. 10 was fitted to all measurements by a least-squares method, resulting

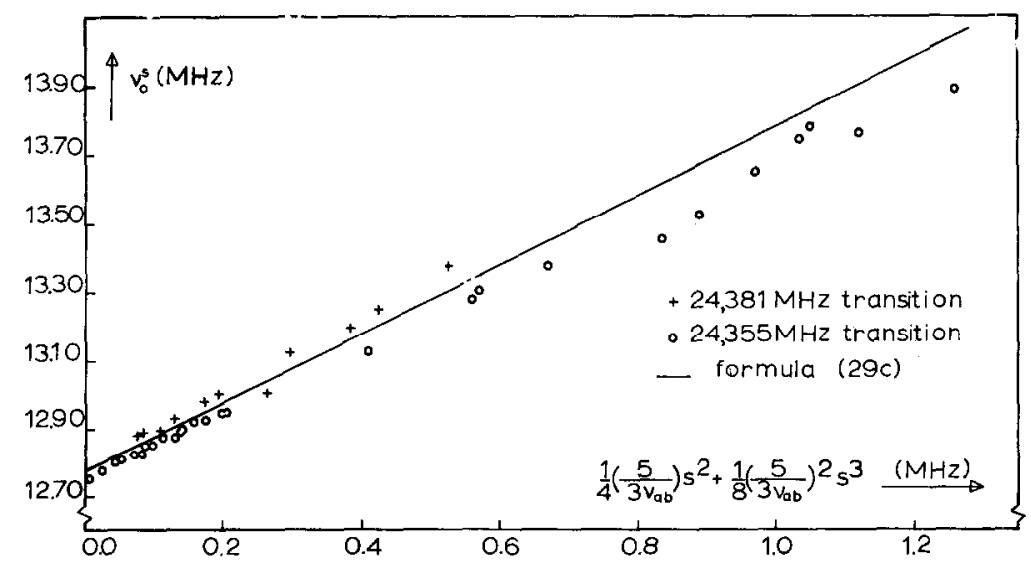

Fig. 10. Plot of the frequency of symmetry $\nu_{0}^{s}$ against the doublet splitting $s$. The straight solid line is a least-squares fit to all measurements + and 0 , which gives $v_{a b}=12.78 \mathrm{MHz}$. This value, in turn, is then used to plot the measurements. 
in a determination of $\nu_{a b}$, the adjustable parameter in eq. (29c). We found:

$$
v_{a b}=12.78 \pm 0.01 \mathrm{MHz} \text {. }
$$

From these experiments it was concluded that, using double-resonance techniques, it is also possible to measure rf transition frequencies as preciscly as microwave absorption-linc frequencies. In fig. 10 data derived from both microwave transitions $a \rightarrow c$ and $b \rightarrow d$ are shown. It is seen that the experimental points deviate systematically from the straight line calculated from eq. (29c), in contradiction to the theory developed in section 2.1. There are two effects that account for this, one valid for the region of small doublet splitting $s$ and the other for the case of large $s$. The first one is due to the fact that the energy levels $(J, M)=(1,0)$ and $(2,0)$ are not disturbed by the rf field because $\mu_{a b}=\mu_{c d}=0$ for $M=0$. Thus the microwave transition $(J, M)=(1,0) \rightarrow(2,0)$ is not influenced by the applied rf field $E_{0}$. But the Stark-modulation voltage used for the detection of microwave absorption lines can be (and was) chosen in such a way that this transition results in a weak signal only (second-order Stark effect!), whereas the transitions $(J, M)=(1, \pm 1) \rightarrow(2, \pm 1)$ are displayed completely. The weak signal from the $(1,0) \rightarrow(2,0)$ transition gives an obvious explanation of the negative absorption peaks observed by Papoular ${ }^{21}$ ). Due to the resulting weak signal at the centre position of the original microwave absorption line, a slightly asymmetric doublet can be observed as an apparent symmetric doublet and thus the required $\nu_{0}^{\mathrm{B}}$ is in error. Further it can be shown that this shift for the $a \rightarrow c$ transition is opposite to that of the $b \rightarrow d$ transition. Numerical calculations on this shift agreed very well with experimental observations at small $s$, which will not be discussed here any further. Fig. 11

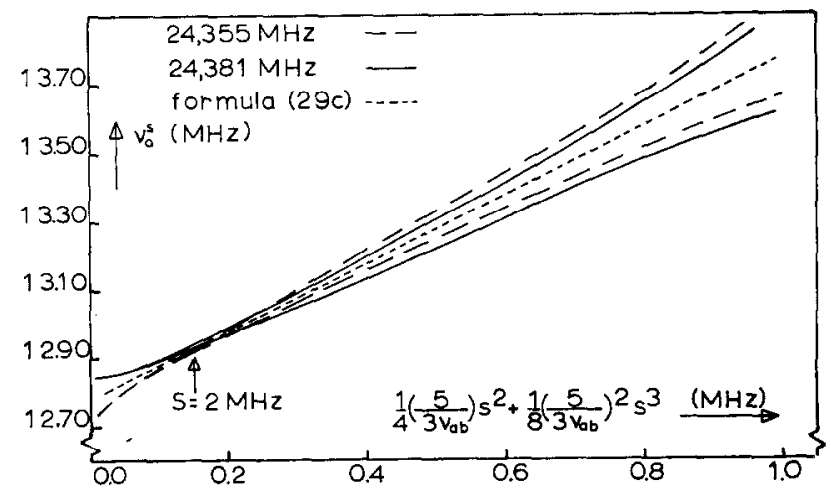

Fig. 11. Sketch of the shifts in the frequency of symmetry $\nu_{0}^{8}$ to be expected as explained in the text. For small $s$ one has $\left(v_{0}^{\mathbf{s}}\right)_{24,355}<\left(v_{0}^{\mathbf{s}}\right)_{24,381}$. For large doublet splitting $s$ either the same inequality holds or the inequality sign should be reversed, depending upon the specific transmission properties of the absorption cell at frequencies in the range from $24,355 \mathrm{MHz}$ up to $24,381 \mathrm{MHz}$. 


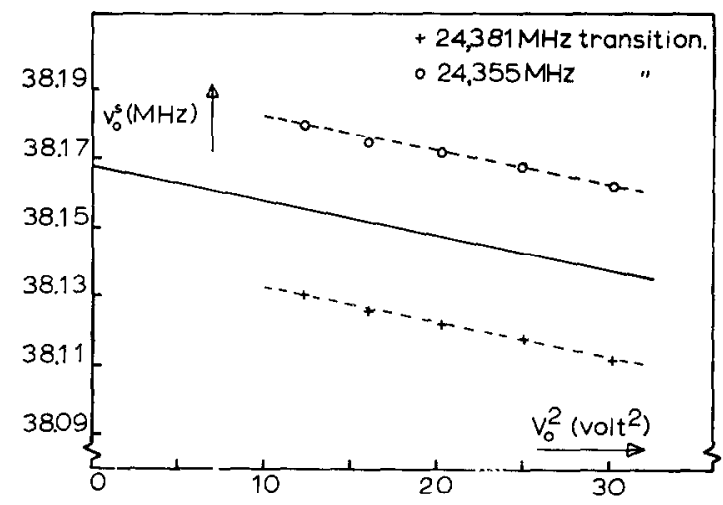

Fig. 12. Plot of the frequency of symmetry $v_{0}^{s}$ for the case $v_{0} \approx v_{c d} \cdot v_{0}^{s}$ is plotted against the square of the measured value of the applied if voltage $V_{0}$, because the rf field strength could not be determined in this case.

gives an illustration of the shifts in $v_{0}^{\mathrm{s}}$ values to be expected for $s \lesssim 2 \mathrm{MHz}$ ( $2 \mathrm{MHz}$ is a representative value: it depends on the linewidth $1 / \tau$ and the Stark-modulation voltage). At large doublet splitting ( $s \gtrsim 2 \mathrm{MHz}$ ) the effect mentioned above should disappear. However, the two components of a doublet are so far apart in frequency, that other difficulties may arise due to frequency-dependent transmission properties of the absorption cell (multiple reflections at both ends of the waveguide). Some possible effects of these reflections on $\nu_{0}^{\mathrm{s}}$ are given in fig. 11 .

The results of double-resonance measurements using an rf signal of $38 \mathrm{MHz}$ in near resonance with $\boldsymbol{v}_{c d}$ are qualitatively in agreement with the predictions of eq. (30a). That means that in this case $\boldsymbol{v}_{0}^{\mathrm{s}}$ should decrease with increasing rf field strength $E_{0}$. Complete splitting of the absorption lines due to the rf field could hardly be observed. The reason is that the length of the absorption cell $(\approx 3 \mathrm{~m})$ is about half the wavelength corresponding to a frequency of roughly $38 \mathrm{MHz}$. Thus, the rf field strength $E_{0}$ is not at all constant along the length of the cell. The observed double-resonance effect is an average over the length of the cell. Some results are shown in fig. 12. The horizontal scale is proportional to $b^{2}$; the slope of the straight lines in fig. 12 cannot be compared with theory because no splitting but only rf voltages have been measured. We conclude that the value of the $J=2 l$-doublet spacing of OCS is:

$$
v_{c d}=38.17 \pm 0.01 \mathrm{MHz} .
$$

Again, the differences in $v_{0}^{8}$ for the two microwave absorption lines are clearly visible. Repeated measurements with an absorption cavity eliminated the problem of cell length and complete doublet splitting showed up. However, the theory given above is not applicable in the latter case: $\mathrm{rf}$ and $\mathrm{mf}$ fields 


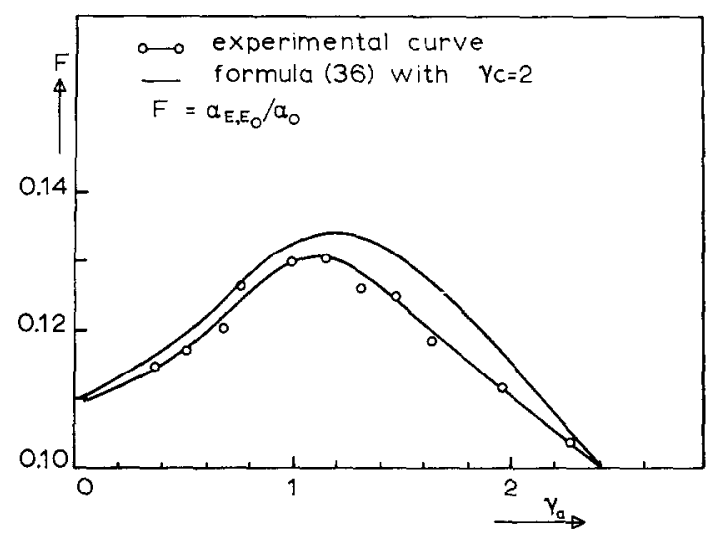

Fig. 13. The measured ratio of the mf absorption coefficient to the mf absorption coefficient in the limit of very weak fields. This ratio was measured at constant $\gamma_{e}$ (mf field strength) as a function of $\gamma_{a}$ (rf field strength).

are not parallel but perpendicular and we have the selection rule $\Delta M= \pm 1$ instead of $\Delta M=0$. Extensions of theory and experiment to this case will be studied in the near future.

3.2. Case B. A second interesting case was the combination of two radiation fields of saturating strength [definition (13)]. Fields of only saturating strength induce no splitting of the corresponding absorption lines. A combination of two frequencies $v$ and $\nu_{0}$ was chosen, both tuned at resonance with the appropriate transitions. The mf field strength was kept at a constant value such that $\gamma_{c} \approx 1$ and the mf frequency was equal to $v_{a c}$ or $\boldsymbol{v}_{b d}$. A saturated absorption line was then observed with the spectrometer described above. When the rf field was applied with increasing strength we first observed an increase of the microwave absorption signal, followed by a rapid decrease as predicted by eq. (37). The calculated curve, eq. (36), together with experimental results is shown in fig. 13. It is seen that the theory of Di Giacomo gives a good description of our results. This suggests the validity of this theory of double resonance for two electromagnetic ficlds, both of saturating strength at most.

Acknowledgements. It is a pleasure to thank Professors C. Th. J. Alkemade and B.R.A. Nijboer and Drs. U. M. Titulaer for valuable discussions and for reading the manuscript. We acknowledge the assistance of Drs. L. van der Hart during the experimental part of this research and we are very greatful to Mr. T. Ram for his technical assistance and preparation of the figures.

This work is part of the research program of the "Stichting voor Fundamenteel Onderzoek der Materie" (F.O.M.) and was made possible by financial 
support from the "Nederlandse Organisatie voor Zuiver Wetenschappelijk Onderzoek" (Z.W.O.).

\section{APPENDIX}

The concepts concerning eq. (18) can be formalized. We shall be dealing here with vectors $a$, consisting of an infinite row of real elements $a_{n}$ :

$$
a=\left(\begin{array}{l}
\vdots \\
a_{-1} \\
a_{0} \\
a_{1} \\
\vdots
\end{array}\right) .
$$

We have the usual definition of the real scalar product of two such vectors:

$$
(p, q)=\sum_{n=-\infty}^{\infty} p_{n} q_{n}
$$

and we have $(p, q)=(q, p)$. We restrict oursclves to all vectors $a$ with $(a, a)<\infty$; they span a linear vector space $R$. Next we define a set of linear operators $a \rightarrow a^{8}$, where $s$ is any integer and $a$ stands for any vector $a \in R$; the definition fixes the $n$th component of $a^{8}$ :

$$
\left(a^{s}\right)_{n}=\left\{\begin{aligned}
a_{s+n} & \text { if } s=\text { even, } \\
(-1)^{n} a_{s-n} & \text { if } s=\text { odd. }
\end{aligned}\right.
$$

Of course we have $a^{0}=a$. Each of these linear operators is a one to one mapping of $R$ on itself. Let $\mathscr{H}$ be the infinite matrix from eq. (18), then eq. (18) can now be rewritten

$$
\mathscr{H} a=L_{a} a \text {. }
$$

Now eq. (19) states that if $a$ is an eigenvector of $\mathscr{H}$ and $(a, a)=1$, the set of vectors

$$
\ldots, a^{-n}, \ldots, a^{-1}, a^{0}, a^{1}, \ldots, a^{n}, \ldots
$$

is an orthonormal set, containing all eigenvectors of $\mathscr{H}$. This set will be complete, i.e. in Dirac's notation:

$$
\sum_{n}\left|a^{n}\right\rangle\left\langle a^{n}\right|=1,
$$

and orthonormality gives (we now have two notations):

$$
\left(a^{k}, a^{l}\right)=\left\langle a^{k} \mid a^{l}\right\rangle=\delta_{k l} .
$$


Eq. (22) is a special case of (A.7) (take $k=0$ and $l=2 s$ ). $A_{p}$ and $C_{p}$ from eq. (24) can be written:

$$
A_{p}=\left(a, c^{2 p+1}\right), \quad C_{p}=\left(a, c^{2 p}\right),
$$

where $a$ and $c$ are the vectors constructed from the $\left\{a_{n}\right\}$ and $\left\{c_{n}\right\}$ defined in the text between eqs. (22) and (23). We have:

$$
\sum_{p}\left(A_{p}^{2}+C_{p}^{2}\right)=\sum_{p}\left(a, c^{p}\right)^{2}=1 .
$$

The second equality in (A.9) is trivial: like the vector set $\left\{a^{n}\right\}$, the set $\left\{c^{n}\right\}$ is a complete orthonormal set of eigenvectors to a matrix $\mathscr{H}^{\prime}$, formed out of $\mathscr{H}$ by the substitutions $\omega_{a b} \rightarrow \omega_{c d} ; b_{a} \rightarrow b_{c}$. So the set $\left\{c^{n}\right\}$ satisfies eqs. (A.6) and (A.7). Thus we have:

$$
1=\langle a \mid a\rangle=\sum_{p}\left\langle a \mid c^{p}\right\rangle\left\langle c^{p} \mid a\right\rangle=\sum_{p}\left(a, c^{p}\right)^{2} .
$$

Finally eq. (27) can be rewritten as:

$$
(a, c)^{2}==\left(a, c^{1}\right)^{2} \text {. }
$$

\section{REFERENCES}

1) Javan, A., Phys. Rev. 107 (1957) 1579.

2) Battaglia, A., Gozzini, A. and Polacco, E., Nuovo Cimento 14 (1959) 1076.

3) Yajima, T. and Shimoda, K., J. Phys. Soc. Japan 15 (1960) 1668.

4) IDi Giacomo, A., Nuovo Cimento 14 (1959) 1082.

5) Di Giacomo, A. and Santucci, S., Nuovo Cimento 633 (1969) 407.

6) Battaglia, A., Di Giacomo, A. and Santucci, S., Nuovo Cimento 43 (1966) 89.

7) Unland, M. L., Weiss, V. W. and Flygare, W. H., J. chem. Phys. 42 (1965) 2138.

8) Cox, A. P., Flynn, G. W. and Wilson, E. B., J. chem. Phys. 42 (1965) 3094.

9) Woods, R. C., Ronn, A. M. and Wilson, E. B., Rev. sci. Instrum. 37 (1966) 927.

10) Auther, S. H. and Townes, C. H., Phys. Rev. 100 (1955) 703.

11) Shimoda, K., J. Phys. Soc. Japan 14 (1959) 954.

12) Kubo, R., Lectures in Theoretical Physics, vol. I (1959), p. 120.

13) Karplus, R. and Schwinger, R., Phys. Rev. 73 (1948) 1020.

14) Shirley, J. H., Phys. Rev. 138 (1965) B979.

15) Cameron, R. H., Duke Math. J. 1 (1935) 356.

16) Morse, P. M. and Feshbach, H., Methods of Theoretical Physics (1953) p. 560.

17) Van Vleck, J. H. and Weisskopf, V. F., Rev. mod. Phys. 17 (1945) 227.

18) Chardon, J. C., Genty, C., Gillet, D., Gire, A., Lomaglio, G. and Mandret, G., CR Acad. Sci. Paris 269 (1969) 1181.

19) Dijkerman, H. A., Thesis, Utrecht (1966).

20) Bloch, F. and Siegert, A., Phys. Rev. 57 (1940) 522.

21) Papoular, A., Phys. Rev. Letters 30A (1969) 412. 